\title{
A COMMON-GATE CMOS INVERTER WITH $n$-CHANNEL AMORPHOUS SILICON THIN-FILM TRANSISTOR FORMED ON A CRYSTALLINE PMOSFET
}

\author{
Heng-Chih Lin, Wen-Jyh Sah and Si-Chen Lee \\ Department of Electrical Enginecring, National Taiwan University, Taipei, Taiwan, R. O. China \\ (Received 18 April 1992; in revised form 5 June 1992)
}

\begin{abstract}
A common-gate complementary metal-oxide-semiconductor (CMOS) inverter consisting of an $n$-channel amorphous silicon $(\mathrm{a}-\mathrm{Si}: \mathrm{H})$ thin-film transistor on top of $1.2 \mu \mathrm{m}$ high $\mathrm{Al}$ gate of the crystalline silicon $p$-channel metal-oxide-semiconductor (PMOS) transistor has been achieved successfully. The success of this inverter demonstrates the feasibility of depositing $3500 \AA$ thick amorphous silicon material on a surface with roughness in the order of $1.2 \mu \mathrm{m}$. It is found that growing an undoped amorphous silicon layer before the deposition of $\mathrm{SiN}_{x}$ insulator is necessary to avoid the permanent destruction of the underlying PMOS due to the stress imposed by the $\mathrm{SiN}_{x}$. The vertical integration of crystalline silicon and amorphous silicon devices to form three-dimensional circuits is a promising technique for future applications in high density memory cell and neural network image sensors.
\end{abstract}

In the advance of ultra-large scaled integrated (ULSI) circuit technology, the size of the individual device has been shrunk continuously. One way to achieve this is to stack the load element on top of the transistor to form a three-dimensional (3D) architecture such as the integration of a thin film transistor (TFT) on top of the metal-oxide-semiconductor (MOS) field effect transistor in $4 \mathrm{Mb} \mathrm{SRAM}$ [1-3]. In addition, in the application of neural network image sensors[4], the amorphous edge detector can be grown directly on the crystalline silicon ULSI circuit, so that the detected edge signals can be processed by the underlying circuit in parallel. In this paper, we present the results on the successful integration of a common-gate complementary metal-oxide-semiconductor (CMOS) inverter which is composed of an amorphous silicon (a-Si:H) TFT on top of a $1.2 \mu \mathrm{m}$ high $\mathrm{Al}$ gate of the crystalline silicon $p$-channel MOS (c-PMOS) transistor.

Figure 1(a) and (b) show the circuit diagram and cross section of the integrated PMOS transistor with an amorphous TFT (a-TFT) as the active driver. The fabrication processes are: the starting silicon wafer is a lightly doped $n$-type substrate. After the standard RCA cleaning process, the $7000 \AA$ field oxide is grown. The first lithographic step is to pattern the source and drain regions, the field oxide on top of these regions is etched off using buffered oxide etch (BOE). Then a thin buffer oxide about $300 \AA$ thick is grown, followed by source and drain implantation with $\mathrm{BF}_{2}^{+}$. The second step is to define the active region. The field oxide in this region is first stripped, then a $400 \AA$ thick gate oxide is regrown. Its quality and uniformity are critical in determining the proper device characteristics. The next step is to open source and drain contact regions. The final step is to evapor- ate and pattern $\mathrm{Al}$ metal followed by annealing at 350 C for $10 \mathrm{~min}$ so that the crystalline PMOS is completed. The gate length and width are 30 and $300 \mu \mathrm{m}$, respectively.

As for the fabrication processes for the upper a-TFT, the first step is the deposition of $200 \mathrm{~nm} \mathrm{Cr}$ and patterned to cover the contact window area. This Cr serves as etching stop because the etching solution of a-Si: $\mathrm{H}$ will also attack underlying $\mathrm{Al}$. Afterwards, a $200 \mathrm{~nm}$ thick a-Si: $i$ layer, $150 \mathrm{~nm} \mathrm{SiN}, 200 \mathrm{~nm}$ a-Si: $\mathrm{H} i$, and $50 \mathrm{~nm} n^{+}$layers are grown successively. The deposition conditions are the following: substrate temperature $250^{\circ} \mathrm{C}$, chamber pressure 0.5 torr, RF frequency $10 \mathrm{MHz}$. The doping of $n^{+}$layer is from $3600 \mathrm{ppm} \mathrm{PH}_{3}$. The first $i$ layer is a buffer layer to separate gate oxide $\mathrm{SiO}_{2}$ and $\mathrm{SiN}_{x}$. It is found that if no buffer layer is inserted between these two materials, there will be a fatal destruction of the $I-V$ characteristics of underlying c-PMOS due to the stress between $\mathrm{SiO}_{2}$ and $\mathrm{SiN}_{r} . \mathrm{SiN}_{x}$ is the gate insulator of the a-TFT. The second $i$ layer serve as channel with the $n^{+}$layer as the contact layer. Then the windows are opened to contact the underlying $\mathrm{Cr}$. Finally the Al layer is evaporated and patterned and the $n^{+}$a-Si: $\mathrm{H}$ layer is then etched away by reactive ion etching (RIE). This completes the entire fabrication process. The gate length and width are 20 and $280 \mu \mathrm{m}$, respectively.

In the integration of the PMOS and top amorphous TFT, a serious problem was encountered. When a $150 \mathrm{~nm}$ thick $\mathrm{SiN}_{x}$ is deposited directly onto the thin $\mathrm{SiO}_{2}$ on top of the drain of the PMOS, the device properties of PMOS are seriously degraded. The common source $I-V$ characteristics of a $n$-channel MOS (NMOS) before and after degradation were shown in Fig. 2(a) and (b). Before deposition of $\mathrm{SiN}_{x}$, 
the NMOS behaves normally with a threshold voltage of $-0.3 \mathrm{~V}$. After deposition of $\mathrm{SiN}_{x}$, a leakage path between $n^{+}$source (drain) and $p^{-}$bulk region was apparently formed so that the NMOS cannot be turned off. This is due to the fact that if the bulksource (drain) $p-n$ junction periphery are not protected by the thick field oxide due to the misalignment in the photolithography process and undercut in the etching process, degradation will occur, which persists even when the $\mathrm{SiN}_{x}$ layer has been etched away. The problem may be due to the stress-induced interface states at the $p-n$ junction periphery. This difficulty is removed by growing a $200 \mathrm{~nm}$ thick undoped a-Si: $\mathrm{H}$ layer before the deposition of $\mathrm{SiN}_{x}$. Figure 2(c) shows the final $I-V$ characteristics of the NMOS after adopting this new process, and no leakage current is observed. The merits of this new process are two-fold: firstly, it prevents the stress on the underlying $\mathrm{SiO}_{2}$. Secondly, the inserted $i$ layer can serve as a protective layer to avoid the transient breakdown of the amorphous TFT. The reason is that under normal operation conditions, the buffer layer is a dielectric insulator. When there is a large voltage pulse applied to the gate, the $i$ layer will absorb most part of the potential drop initially because it acts as a resistor in series with the $\operatorname{SiN}_{x}$ capacitor. This prevents the permenent breakdown of the $\operatorname{SiN}_{x}$ gate.

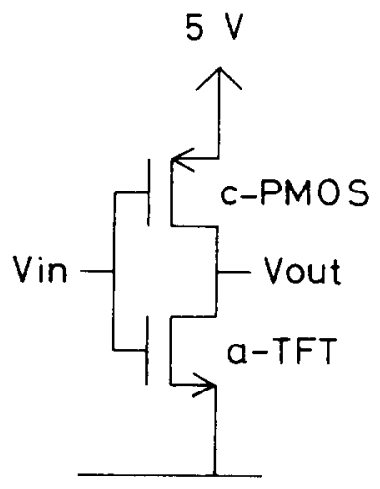

(a)

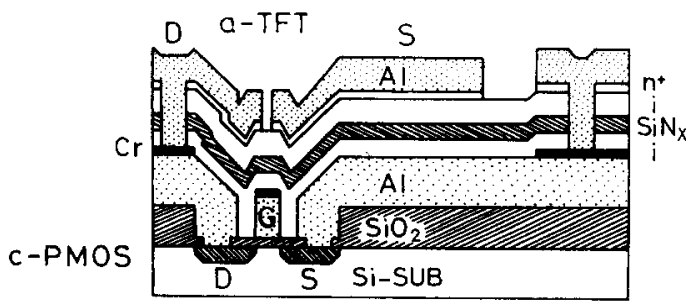

(b)

Fig. 1. (a) The circuit diagram and (b) cross section of the integrated common gate $\mathrm{CMOS}$ inverter consisting of a crystalline PMOS and amorphous TFT.
Figure 3(a) shows the common-source $I-V$ characteristics of underlying PMOS with gate bias varying from -1 to $-6 \mathrm{~V}$. The maximum field-effect mobility in the saturation region is $39 \mathrm{~cm}^{2} / \mathrm{V}-\mathrm{s}$. The reason for the low field-effect mobility is probably that the nonself-align processes introduce a large source series resistance. Figure 3(b) shows the transfer $I_{\mathrm{D}}$ vs $V_{\mathrm{GS}}$ curve of this PMOS. The threshold voltage is $-1.2 \mathrm{~V}$ and the transconductance at $1 \mathrm{~mA}$ is $1.5 \mathrm{mS} / \mathrm{mm}$. Figure 4(a) shows the common source $I-V$ characteristics of the top amorphous TFT with the gate bias as the independent parameter.

(a)
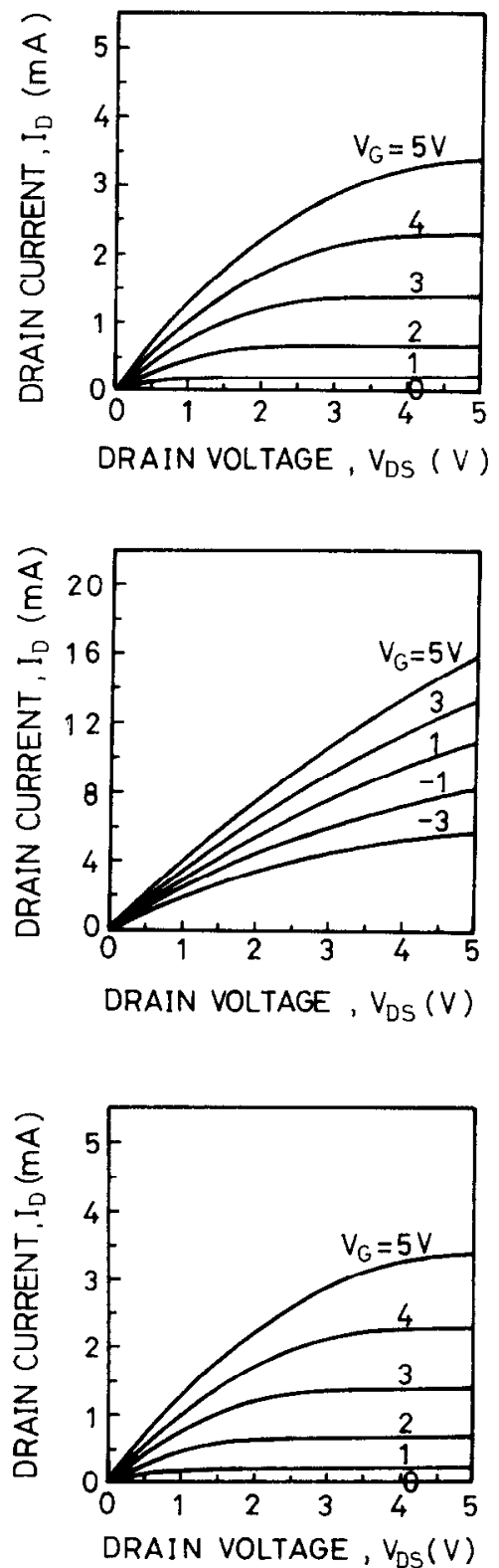

Fig. 2. (a) Original and (b) degraded $I-V$ charcteristics of crystalline NMOS before and after the deposition of $\mathrm{SiN}$. on $\mathrm{SiO}_{2}$, respectively. (c) The $J-V$ characteristics of underlying NMOS after the insertion of amorphous layer between $\mathrm{SiN}_{x}$ and $\mathrm{SiO}_{2}$ layer 
Figure 4(b) displays the transfer $I_{D}$ vs $V_{G S}$ plot of this a-TFT. The on-off ratio exceeds four orders of magnitude and the threshold voltage is $1.5 \mathrm{~V}$. The field effect mobility obtained from the $I_{\mathrm{D}}$ vs $V_{\mathrm{DS}}$ plot is $0.23 \mathrm{~cm}^{2} / \mathrm{V}-\mathrm{s}$, which is typically of a-SiN $\mathrm{S}_{x}$ gate TFT. The gate is shorted to the drain during the measurement. Figure 5 shows the transfer curve of this CMOS inverter. For gate voltage $V_{G S}$ smaller than $2.7 \mathrm{~V}$, the PMOS is on and TFT is off, so that the applied voltage $5 \mathrm{~V}$ is fed through the PMOS and appears at the output. When $V_{G S}$ exceeds $2.7 \mathrm{~V}$, PMOS is turned off, while the TFT is turned on. The output voltage is driving to below $0.2 \mathrm{~V}$.

In conclusion, the integration of the common gate CMOS inverter which consists of an amorphous TFT directively grown on top of the crystalline PMOS

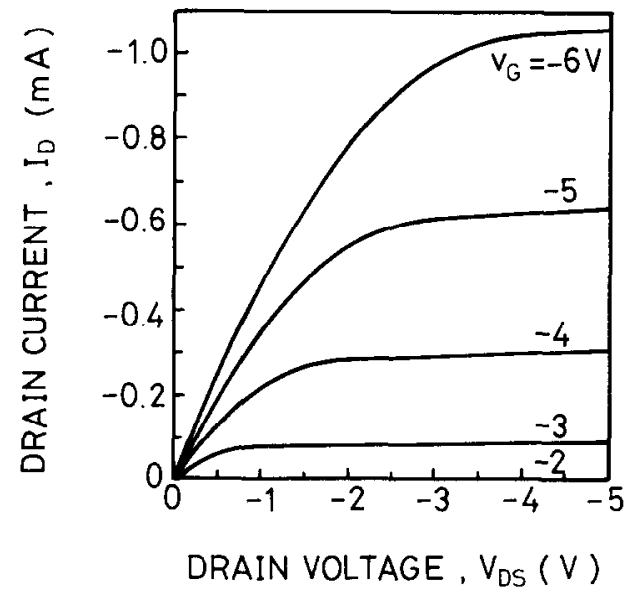

(a)

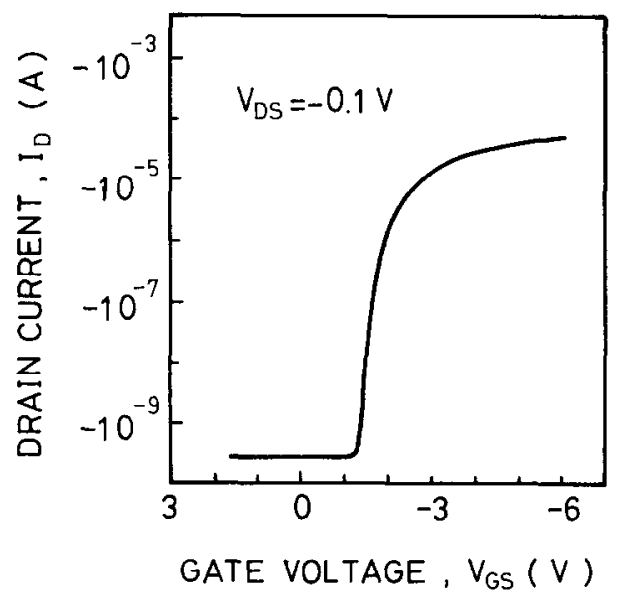

(b)

Fig. 3. (a) The common source $I-V$ characteristics of PMOS in the integrated inverter. The gate bias $V_{\mathrm{G}}$ varies from -1 to $-6 \mathrm{~V}$. (b) The transfer $I_{\mathrm{D}}$ vs $V_{\mathrm{GS}}$ characteristics of c-PMOS in the integrated circuit. $V_{D S}$ is fixed at $-0.1 \mathrm{~V}$.

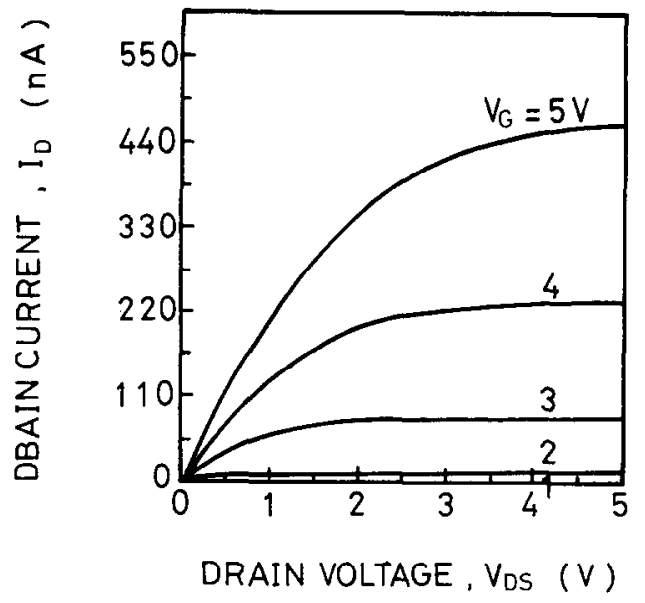

(a)

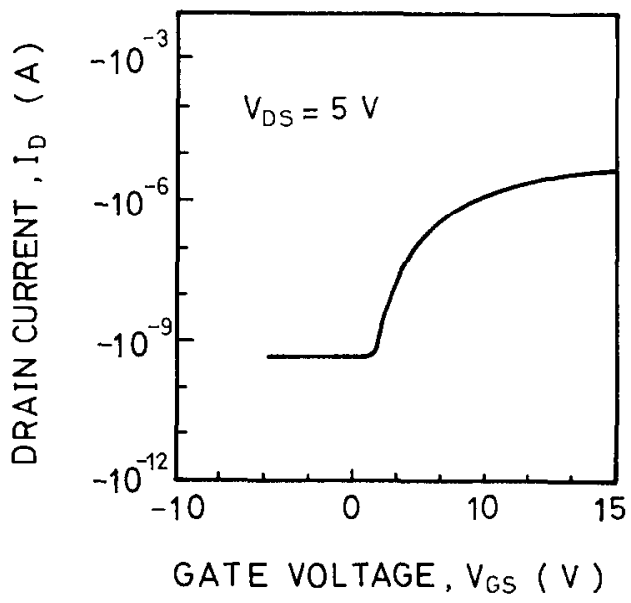

(b)

Fig. 4. (a) The $I-V$ characteristics of amorphous TFT in the integrated inverter. The gate bias $V_{\mathrm{GS}}$ varies from -3 to $5 \mathrm{~V}$ (b) The transfer $I_{\mathrm{D}}$ vs $V_{\mathrm{GS}}$ characteristics of amorphous TFT in the integrated circuit. $V_{\mathrm{DS}}$ is fixed at $5 \mathrm{~V}$.

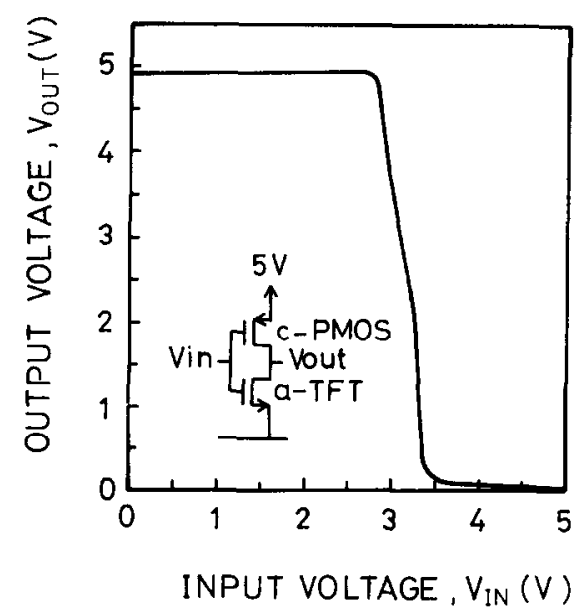

Fig. 5. The transfer curve of the integrated CMOS inverter. 
over $1.2 \mu \mathrm{m}$ thick $\mathrm{Al}$ gate has been successfully achieved. Furthermore, a new technique by inserting a buffer $i$ layer between $\mathrm{SiO}_{2}$ and $\mathrm{SiN}_{4}$ layer is proposed to solve the stress problem and preserved the normal performance of the crystalline MOSFET.

Acknowledgements-.-This work is supported by the $\mathrm{Na}$ tional Science Council of the Republic of China under contract No. NSC 80-0417-E002-04.

\section{REFERENCES}

1. A. O. Adan, K. Suzuki, H. Shibayama and R. Miyake, IEEE Symp. on VLSI Technology, pp. 19-20 (1990).

2. Y. Uemoto, E. Fujii, A. Nakamura and K. Senda, IEEE Symp. on VLSI Technology, pp. 21-22 (1990).

3. M. Kinugawa, M. Kakumu, T. Yoshida, T. Nakayama, S. Morita, K. Kubota, F. Matsuoka, H. Oyamatsu, $\mathrm{K}$. Ochii and K. Macguchi, IEEE Symp. on VLSI Technology, p. 23 (1990).

4. W. J. Sah, S. C. Lee, H. K. Tasi and J. H. Chen, Appl. Phys. Lett. 56, 2539 (1990). 